\title{
What Humeral Position is Better to Prevent Shoulder Impingement during Arm Elevation?
}

\author{
Mi-jeong Kim, PT, MSc${ }^{1}$; Jong-hyuck Weon, PT, Ph.D² \\ Department of KEMA Therapy, Graduate School of Humanities Industry, Joongbu University, Geumsan, South Korea \\ Department of Physical Therapy, College of Tourism \& Health Science, Joongbu University, Geumsan, South Korea
}

Background Secondary impingement can be caused by an imbalance in the force couple or coactivation of the deltoid and rotator cuff, as well as by an imbalance in the scapular upward rotators.

Purpose The purpose of this study was to evaluate the effects of humeral position and shoulder flexion angle on the activity of the scapular upward rotators, infraspinatus, and deltoid during arm elevation.

Study design Cross-sectional comparative study.

Methods Twenty-three healthy participants were included in this study. Electromyography (EMG) electrodes were attached to the upper trapezius, lower trapezius, serratus anterior, infraspinatus, anterior deltoid, and middle deltoid. The participants were seated on a chair with no arm rest and instructed to maintain the trunk in an upright position. They were then asked to elevate the arm until it reached a bar set at 90 degrees and 120 degrees elevation. The movement was performed in the scapular plane to ensure shoulder joint stability. Participants were then asked to grasp a 2-kg dumbbell and maintain arm elevation for 5 seconds in the palm-down, neutral, and palm-up position. Muscle activity was measured during maximal voluntary isometric contraction (MVIC), and \%MVIC values were obtained. Two-way repeated-measured analysis of variance was used for the analysis; when significant differences were found in the \%MVIC values of each muscle according to the arm elevation position, post hoc paired-sample $t$-test with Bonferroni correction were performed.

Results The EMG activity of the upper trapezius, lower trapezius, serratus anterior, infraspinatus, anterior deltoid, and middle deltoid varied significantly according to the shoulder flexion angle (all $p<0.05$ ) and according to the humeral position (all $p<0.05$ ) in all muscles except the lower trapezius.

Conclusions We recommend that consideration be given to the humeral position and shoulder flexion angle during the performance of strengthening exercises for the scapular upward rotators and shoulder external rotators.

Key words Humeral position; Scapular upward rotator; Shoulder dysfunction; Shoulder external rotation; Shoulder impingement syndrome.

\section{INTRODUCTION}

There are two causes of shoulder impingement syndrome (SIS), first described by Neer: the primary cause being the
JMST

2020; 4(1): 30-39

Published Online Jun 30, 2020

pISSN 2635-8573

eISSN 2635-8581

Article History

Received 06 Jun 2020

Revised 08 Jun 2020

(1st)

Accepted 08 Jun 2020

\section{CONTACT}

jhweon@joongbu.ac.kr Jong-hyuck Weon, Department of Physical Therapy, College of Tourism \& Health Science, Joongbu University, Geumsan, South Korea

This is an Open-Access article distributed under the terms of the Creative Commons Attribution Non-Commercial License (http://creativecommons. org/licenses/by-nc/4.0) which permits unrestricted non-copermits unrestricted non-comercial use, distibution, and reproduction in any medium, provided the origina work is properly cited. narrowing of the interarticular space due to soft tissue infection or bone growth and the secondary cause being the upward movement of the humeral head because of the weakness of the muscles or the imbalance of the force 
among the muscles around the shoulders. ${ }^{1,2,3}$ Secondary impingement is mainly caused by the force imbalance among the shoulder muscles generating the force couple. The imbalance or co-activation of the deltoid and rotator cuff muscles and the imbalance of the scapular upward rotators are known to be the main causes of SIS., ${ }^{4,5,6}$

When an imbalance occurs between the force couple of the deltoid and rotator cuff muscles, the subacromial space narrows, and the humeral head slides upward while rising the arm, causing the impingement of the acromion and humeral head. ${ }^{7}$ The scapular downward rotation syndrome, which lacks the scapular upward rotation because of the predominance and shortening of the downward rotators of the scapula, may also cause SIS. ${ }^{8,9}$ In addition, people with SIS symptoms had increased upper trapezius action and reduced lower trapezius and serratus anterior action than normal subjects when elevating their arms, thus resulting in the decreased upward rotation and increased scapular anterior tilt. ${ }^{8,10,11}$ These factors reduce the space between the acromion and humerus, resulting in SIS. The weakening of the external rotators of the glenohumeral joint may also cause the impingement of the greater tubercle of the humerus and the acromion during arm elevation. Therefore, to prevent secondary shoulder impingement, ${ }^{10,11,12}$ it is important to activate the weakened scapular upward rotators and rotator cuff muscles so that the subscapular articular cavity is properly maintained during arm movement. ${ }^{6,13,14}$

Among the scapular upward rotators, the weakened muscles are mainly the serratus anterior and lower trapezius muscles. ${ }^{10,15,16,17,18}$ Exercise methods for strengthening the weakened serratus anterior and lower trapezius muscles include the push-up plus exercise, protraction exercise, prone arm lift exercise, and modified cobra exercise. ${ }^{19,20,21}$ Methods for resolving the imbalance between the deltoid and rotator cuff muscles are as follows: to increase the length of the shortened deltoid muscle and decrease the activity of the deltoid muscle and to activate the rotator cuff muscle. ${ }^{8,22,23}$ To do so, Sahrmann proposed to reduce the load on the deltoid by bending the elbow joint during arm elevation. ${ }^{8}$ Huston and Adam suggested methods for activating the rotator cuff muscle by rotating the upper arms inward or outward. In addition, a method for activating the infraspinatus and teres minor muscles (both of which are rotator cuff muscles) by rotating the upper arm outward and elevating arm through wall sliding with the ulnar side of the hand was introduced. ${ }^{8,24}$

Previous studies investigated various factors affecting muscle activity as well as exercise methods for strengthening the scapular upward rotators or the rotator cuff muscles. For example, it was found that the muscle activity of the rotator cuff muscles changed depending on the head position (forward vs. neutral head position) or visual feedback. ${ }^{9,25}$ It was also reported that the muscle activity of the scapular muscles varied depending on the weight loaded on the hand, grip strength, and the angle of bending of the glenohumeral joint. ${ }^{10,13,26,27}$

According to Xavier et al., the scapular upward rotation was increased and the mobility of the glenohumeral joint was decreased during the full-can test (arm elevation with the thumb pointing upward) compared to during the emptycan test (arm elevation with the thumb pointing downward). ${ }^{28}$ The internal or external rotation of the glenohumeral joint was suggested as a way to activate the rotator cuff muscles. ${ }^{8,23}$ These results indicate that the muscle activity of the scapular upward rotator and rotator cuff muscles is affected by the upper arm positions.

Muscle activity of the scapular upward rotators, rotator cuff muscles, and deltoid muscles is an important factor for SIS. ${ }^{4,8,10,23}$ Nevertheless, to our knowledge, no studies have focused on the muscle activity of the scapular upper rotator and rotator cuff muscles according to the position of the upper arm. Therefore, this study aimed to investigate the effect of upper arm position and the shoulder flexion angle on the activity of the scapular upward rotators, infraspinatus, and deltoid muscles, which are the major muscles associated with SIS.

\section{METHODS}

\section{Participants}

G*Power software (version 3.1.2) was used to estimate necessary sample size. Sample size was calculated a priori for a power of 0.95 , and effect size of 0.80 , and alpha level of 0.05 . This calculation indicated that the necessary sample size was 16 subjects for the study. 23 healthy adults at the Joongbu University participated in this study, and were selected according to the selection criteria. The purpose, method, and procedure of this study were explained to each subject, and they voluntarily participated in the experiment after providing written informed consent. This study was approved by the Institutional Review Board of the Joongbu University.

In this study, the following participants were excluded: those who expressed pain in the past or during clinical examination, those who had functional issues due to restricted shoulder joint motion, those who experienced instability in the shoulder joint during activities of daily living, those who had neurological symptoms around their necks, and those who had experienced numbness or loss of sensation in the upper limb in the six months before the 
experiment. The general characteristics of the subjects are shown in Table 1.

\section{Experimental instruments}

The muscle activity of each muscle was measured using surface electromyography (Noraxon TeleMyo 2400 system, Noraxon Inc, Scottsdale, AZ, USA). Noraxon MyoResearch $1.06 \mathrm{XP}$ was also used to collect and analyze the muscle activity data of the muscles being assessed. Sampling of the electromyogram signal was set to $1,000 \mathrm{~Hz}$, and a band pass filter of $10-450 \mathrm{~Hz}$ was applied to remove the electrocardiogram signal, which is a biological signal noise.

\section{Procedures}

The participants underwent depilation and keratin removal for the attached part before application of the electromyographic electrodes ( $\mathrm{Ag} / \mathrm{AgCl}$ surface electrodes). Their skin was cleaned with alcohol using cotton, and the electrodes were attached according to the standardized position for each muscle. The gap between the electrodes was fixed at 2 $\mathrm{cm}$, and they were attached parallel to the direction of muscle fibers. ${ }^{29}$ The electromyographic electrodes were attached to the upper trapezius, lower trapezius, serratus

Table 1. General characteristics of subjects

$(n=23)$

\begin{tabular}{cc}
\hline Variable & Mean $\pm \mathrm{SD}^{\mathrm{a}}$ \\
\hline Age $(\mathrm{yrs})$ & $22.91 \pm 1.53$ \\
Height $(\mathrm{cm})$ & $163.96 \pm 9.42$ \\
\hline Weight $(\mathrm{kg})$ & $57.35 \pm 10.97$ \\
\hline
\end{tabular}

${ }^{\mathrm{a}}$ Mean \pm standard deviation. anterior, infraspinatus, anterior deltoid, and middle deltoid muscles. The electrodes of the upper trapezius were attached parallel to the direction of the muscle fibers at the middle point between the spinous process of the seventh cervical vertebra and the posterolateral acromion. The electrodes of the lower trapezius were attached to the middle part of the muscle located at an angle of $55^{\circ}$ from the lower angle of the scapula.

The electrodes of the serratus anterior were attached to the parallel muscle of the serratus anterior, which is located at the same height as the inferior angle of the scapula, in front of the latissimus dorsi muscle under the axilla. The electrodes of the infraspinatus muscle were attached parallel to the muscle fibers at the midpoint of the infraspinatus fossa. ${ }^{29}$ The electrodes of the anterior deltoid muscle were attached to the muscle belly of one finger based on the acromion while comfortably lowering their arms. The electrodes of the middle deltoid muscles were attached parallel to the direction of the muscle fibers at the most convex point on the line extending the acromion and lateral epicondyle of the cubitus (Figure 1). ${ }^{30}$

The experiment was conducted by two measurers who had at least three years of clinical experience in physical therapy. One measurer operated the computer, and the other controlled the participants' position and experimental process and did not allow the participants to know the data of their muscle activity displayed on the computer. The two measurers thoroughly understood all the procedures and made it impossible for the measurers and participants to predict the test results and know the meaning of the test results. The participants were given preliminary experiments with detailed explanations of measurement positions and
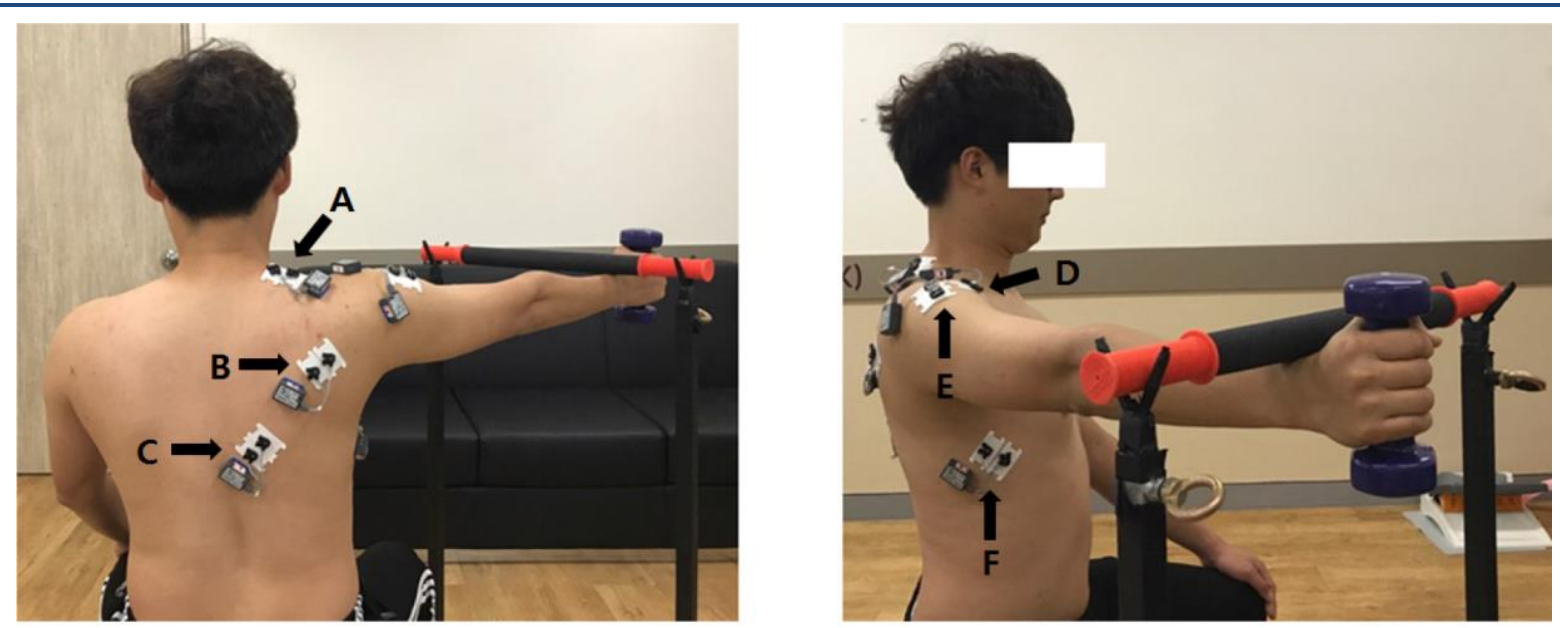

Figure 1. Location of EMG electrode for shoulder girdle muscles (A, upper trapezius; B, infraspinatus; C, lower trapezius; D, anterior deltoid; E, middle deltoid; F, serratus anterior). 
methods so that they could become familiar with the experiment. The participants were assessed in a random order. The experiment was performed in a sitting position on a chair without armrests, and during the experiment, they maintained the correct sitting position.

The EMG signals collected in the following positions: palm down (PD) position, neutral position (NT), and palm up (PU) position with a $2 \mathrm{~kg}$ dumbbell on hand while sitting. The participants raised their hands up to the pre-set targetbar at the angle of $90^{\circ}$ and $120^{\circ}$ with the above positions and maintained their position for $5 \mathrm{sec}$. At this time, the movement of the arm was generated in the scapular plane considering the stability of the shoulder joint (Figure 2) ${ }^{31}$ The experiment was carried out three times with two flexion angles of the shoulder joint $\left(90^{\circ}, 120^{\circ}\right)$ and in three positions (PD, NT, PU). Between two consecutive measurements, a two-minute rest was allowed to prevent muscle fatigue.

\section{Statistical analysis}

The Kolmogorov-Smirnov test was employed to assess normality of the distribution. The data collected through the experiments were analyzed using SPSS ver. 21 for Windows. According to the upper arm position and shoulder flexion angle, a two-way repeated ANOVA was used to determine the change in muscle activity of the shoulder muscles, and the significance level was set at 0.05 . When there was a significant difference in this analysis, post-hoc analysis was performed using the paired-sample $t$-test with Bonferroni correction. The significance level at this time was 0.017 $(0.05 / 3)$.

\section{RESULTS}

Table 2, Table 3, and Figure 3 show the change in the muscle activity of the upper trapezius, lower trapezius, serratus anterior, infraspinatus, anterior deltoid, and middle

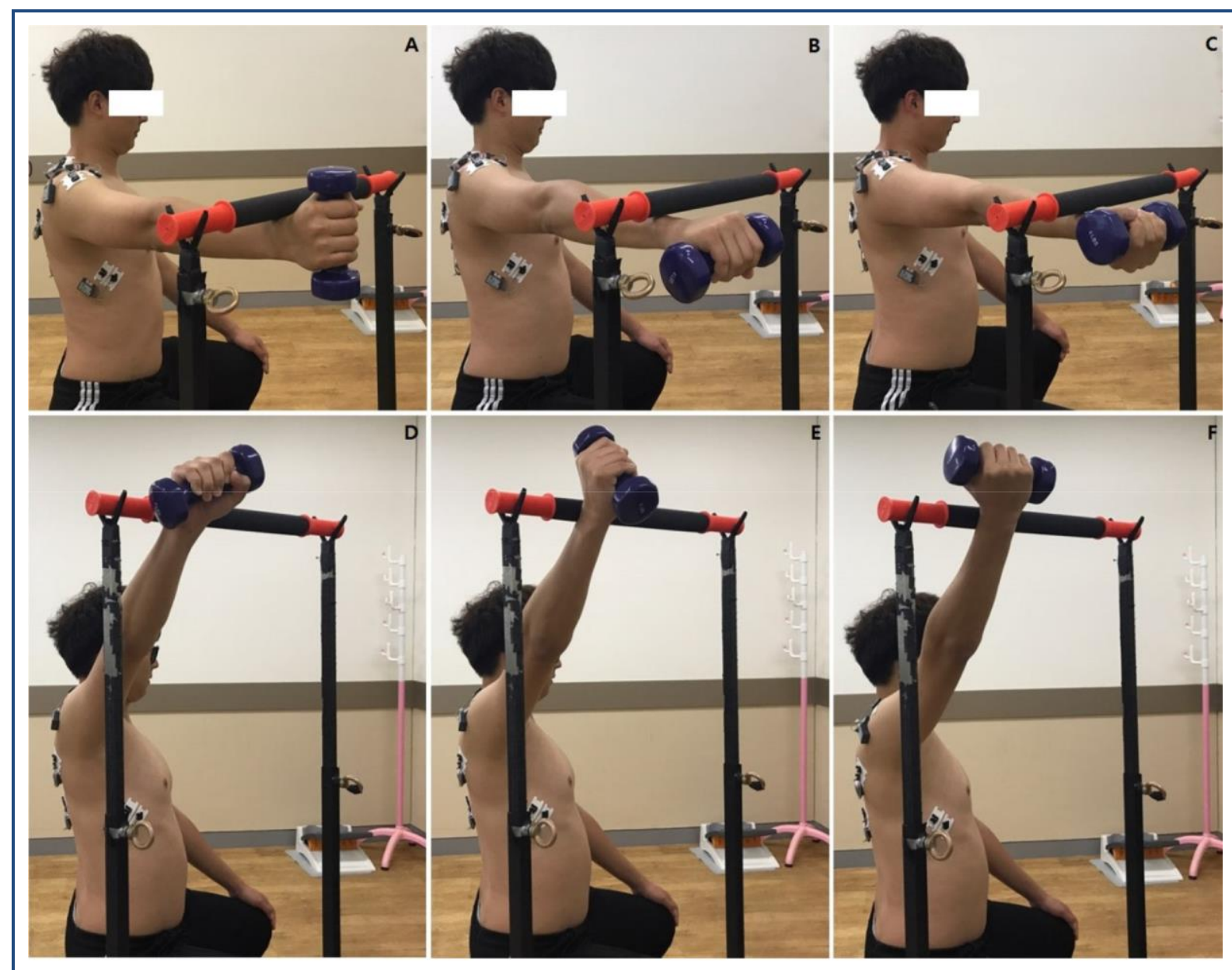

Figure 2. Arm elevation in three humeral positions (A, $90^{\circ}$ neutral position; B, $90^{\circ}$ palm down position; C, $90^{\circ}$ palm up position; $\mathrm{D}, 120^{\circ}$ palm down position; E, $120^{\circ}$ neutral position; F, $120^{\circ}$ palm up position). 
Table 2. Muscle activity in three humeral position at $90^{\circ}$ and $120^{\circ}$ shoulder flexion

$(n=23)$

\begin{tabular}{ccccc}
\hline Angle & Muscles & PD & NT & PU \\
\hline & UT & $31.84 \pm 20.54^{\mathrm{a}}$ & $29.24 \pm 19.01$ & $37.70 \pm 21.25$ \\
LT & $47.97 \pm 18.36$ & $48.02 \pm 19.74$ & $48.79 \pm 21.78$ \\
90 & $35.30 \pm 9.14$ & $33.42 \pm 8.57$ & $48.64 \pm 12.84$ \\
& IS & $40.74 \pm 20.61$ & $39.58 \pm 20.79$ & $50.78 \pm 17.65$ \\
& AD & $48.30 \pm 27.34$ & $48.86 \pm 32.49$ & $71.50 \pm 48.72$ \\
MD & $35.71 \pm 18.79$ & $30.69 \pm 20.85$ & $31.92 \pm 19.87$ \\
UT & $49.58 \pm 32.80$ & $47.86 \pm 32.91$ & $57.36 \pm 30.84$ \\
LT & $40.33 \pm 16.47$ & $34.97 \pm 11.89$ & $40.66 \pm 13.98$ \\
SA & $46.63 \pm 9.85$ & $45.84 \pm 13.84$ & $76.90 \pm 38.94$ \\
& IS & $44.36 \pm 20.59$ & $45.05 \pm 22.32$ & $59.76 \pm 21.25$ \\
AD & $56.36 \pm 39.16$ & $60.89 \pm 52.89$ & $77.16 \pm 46.72$ \\
MD & $61.84 \pm 34.77$ & $53.67 \pm 36.64$ & $54.43 \pm 36.49$ \\
\hline
\end{tabular}

${ }^{\mathrm{a}} \mathrm{Mean} \pm$ SD.

Abbreviation; PD, palm down; NT, neutral; PU, palm up; UT, upper trapezius; LT, lower trapezius; SA, serratus anterior; IS, infraspinatus; $\mathrm{AD}$, anterior deltoid; $\mathrm{MD}$, middle deltoid.

Table 3. Significance of EMG activity of scapular upward rotators and shoulder muscles on humeral position and shoulder flexion angle

\begin{tabular}{cccc}
\hline Muscles & Variables & $F$-value & $p$-value \\
\hline \multirow{2}{*}{ UT } & Angle & 47.76 & $.000^{*}$ \\
& Arm position & 20.09 & $.000^{*}$ \\
& Anglexarm position & 0.69 & 0.51 \\
LT & Angle & 11.72 & $.002^{*}$ \\
& Arm position & 2.46 & 0.11 \\
Anglexarm position & Angle & 2.87 & 0.08 \\
& Arm position & 29.79 & $.000^{*}$ \\
& Anglexarm position & 16.65 & $.000^{*}$ \\
& Angle & 2.55 & 0.10 \\
& Arm position & 10.79 & $.003^{*}$ \\
& Anglexarm position & 7.45 & $.004^{*}$ \\
& Angle & 2.90 & 0.08 \\
& Arm position & 8.87 & $.007^{*}$ \\
& Anglexarm position & 25.82 & $.000^{*}$
\end{tabular}

${ }^{*} p<0.017$.

Abbreviation; UT, upper trapezius; LT, lower trapezius; SA, serratus anterior; IS, infraspinatus; AD, anterior deltoid; MD, middle deltoid. 


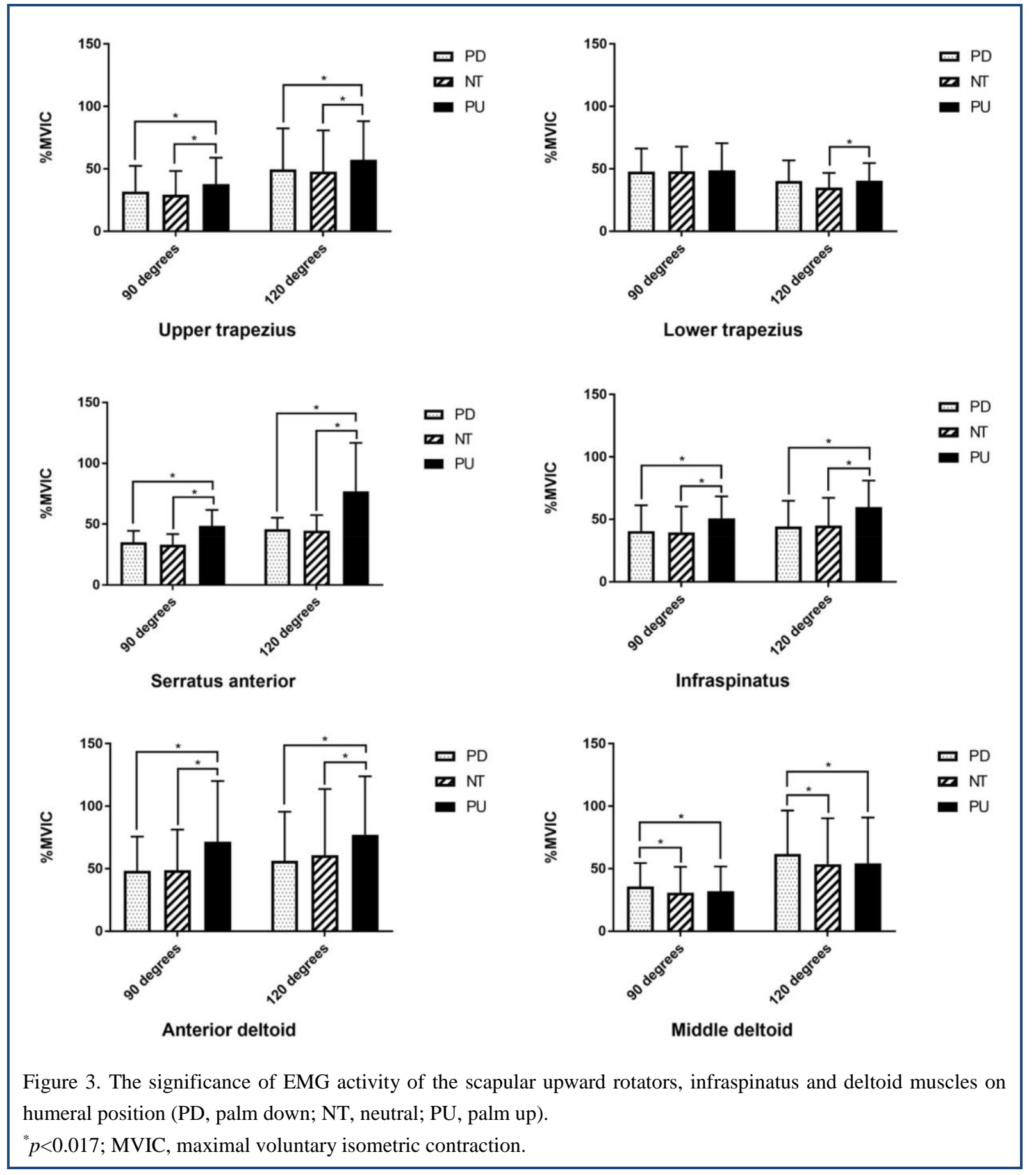

deltoid muscles according to the upper arm position and the flexion angle of the shoulder joint during arm elevation.

For the all muscles of upper trapezius, lower trapezius, serratus anterior, infraspinatus, anterior deltoid, and middle deltoid, the main effects were significant for shoulder flexion angle $(\mathrm{p}<0.05)$. And for the all muscles except lower trapezius, the main effects were significant for upper arm position $(p<0.05)$. There was also no interaction between the two factors in all muscles $(p>0.05)$.

According to the post-hoc analysis, muscle activity of the upper trapezius and serratus anterior muscles in the PU position were significantly higher than the other humeral positions at both angles $(p<0.017)$. And muscle activity at the $120^{\circ}$ flexion angle in all three humeral positions showed significantly higher value than $90^{\circ}$ flexion angle $(p<0.017)$. Muscle activity of the lower trapezius muscle at the PU position was significantly higher than the other two humeral positions only at a $120^{\circ}$ flexion angle $(p<0.017)$.

In the PU position, the muscle activity of the infraspinatus and anterior deltoid muscles was significantly higher 
than the other two positions at both angles $(p<0.017)$, and only in the PU position, it showed a significantly higher muscle activity at a $120^{\circ}$ flexion angle than the a $90^{\circ}$ flexion angle $(p<0.017)$. The muscle activity of middle deltoid muscle displayed a significantly higher in the PD position compared to the other two positions at both angles $(p<0.017)$, and showed significantly higher values at a $120^{\circ}$ flexion angle compared to a $90^{\circ}$ flexion angle in all three positions $(p<0.017)$.

\section{DISCUSSION}

This study aimed to investigate the effect of upper arm position and shoulder flexion angle on the activity of major muscles associated with SIS during arm elevation. The muscle activity of the scapular upward rotators, infraspinatus, and deltoid muscles was significantly different according to the upper arm position and the shoulder flexion angle $(p<0.05)$. There was a significant difference in muscle activity of upper trapezius, serratus anterior, infraspinatus, anterior deltoid, and middle deltoid muscles except for the lower trapezius muscle depending on the upper arm position $(p<0.05)$. There was also a significant difference in muscle activity of all muscles according to the flexion angle of the shoulder joint $(p<0.05)$.

All muscles except for the lower trapezius showed higher muscle activity at $120^{\circ}$ depending on the arm elevation angle $(\mathrm{p}<0.017)$. This is because of the length-tension relationship..$^{32}$ The scapular upward rotators, infraspinatus, anterior deltoid, and middle deltoid muscles are shortened at $120^{\circ}$ rather than $90^{\circ}$, thereby showing a higher muscle activity. Previous studies on shoulder muscles also showed increases in muscle activity as the flexion angle of the shoulder joint increased. ${ }^{4,10,13,33}$ In this study, there was no significant difference in the lower trapezius muscle activity according to the angle. The lower trapezius muscle activity sharply increased in the end range during arm elevation, thus resulting in a small difference between the $90^{\circ}$ and $120^{\circ}$ bending angles of the shoulder joint. ${ }^{34}$

In this study, muscle activity of the upper trapezius and serratus anterior muscles was significantly higher value in the PU position compared to NT and PD positions at both $90^{\circ}$ and $120^{\circ}$ angles $(p<0.017)$. And there was no significant difference between the NT and PD positions in this regard. There are two reasons for the muscle activity being significantly higher in the PU position than in the NT and PD positions.

First, this may have resulted from changes in the mobility of the scapula and the glenohumeral joint due to the upper arm position. ${ }^{28}$ To obtain the PU position, an external rotation of the glenohumeral joint occurs, and the contraction of the infraspinatus and teres minor muscles that occurs at this time limits the mobility of the glenohumeral joint, resulting in an increase in the movement of the scapula. Consequently, the muscle activity of the upper trapezius and serratus anterior muscles may have been further increased due to the length-tension relationship. ${ }^{8,11}$

Second, the passive tension of the teres major muscles may have affected the result. Passive insufficiency of movement due to the teres major muscles may occur while raising the glenohumeral joint in the external rotation to achieve the PU position, thereby acting as a resistance to the scapular upward rotators. ${ }^{8,11}$

In addition, the muscle activity of all the muscles measured in this study did not show a significant difference between the NT and PD positions at both angles; this may be due to the compensation of the forearm and elbow joint. To obtain the PU position, the external rotation of the humerus is essential, but the humerus position in NT and PD is greatly affected by the pronation or supination position of the forearm, thereby leading to no significant difference.

The muscle activity of the infraspinatus was also significantly higher in the PU position than in the NT and PD positions $(p<0.017)$, which may be the inevitable result of the external rotation of the glenohumeral joint to achieve the PU position.

The muscle activity of the anterior deltoid muscle in the PU position was a significantly higher value compared to the NT and PD positions $(p<0.017)$. And the middle deltoid muscle activity was significantly higher in the PD position than in the NT and PU positions ( $p<0.017)$. These observations may have resulted from the change in the direction of motion and the arrangement of muscle fibers by the external rotation of the glenohumeral joint in the PU position.

In this study, the muscle activity of the middle deltoid muscle was significantly increased in the PD position than in the NT and PU positions ( $p<0.017)$. When the action of the middle deltoid muscles is dominant over that of the rotator cuff muscles, the humerus head easily moves upward, causing shoulder impingement. ${ }^{28}$ In the PD position, the greater trochanter of the humerus moves in front of the shoulder, and the space under the shoulder becomes narrower, thereby increasing the possibility of shoulder impingement due to the shearing force. ${ }^{7}$ Therefore, to prevent shoulder impingement during arm elevation, it is preferable to assume the PU position wherein the middle deltoid muscle activity is lower than it is in the PD position and the greater trochanter of the humerus rotates outward.

The serratus anterior is the muscle that rotates the scapula upward, and when the muscle activity is decreases, the 
upward rotation of the scapula becomes difficult and causes shoulder dysfunction, such as winging and SIS. ${ }^{35,36}$ Strengthening the serratus anterior muscle is important to address abnormal scapula movement and prevent shoulder dysfunction. ${ }^{10,18,20,37,38}$ According to the results of this study, the muscle activity of the serratus anterior muscle was significantly higher at $120^{\circ}$ than at $90^{\circ}$ in the $\mathrm{PU}$ position than in the PD and NT positions. Therefore, elevating the arm at an angle of $120^{\circ}$ and maintaining the position may be suggested as an exercise for increasing the muscle activity of the serratus anterior.

There are some limitations of this study. First, this study was carried out on healthy adults who had no abnormality in shoulder function; thus, its applicability to individuals with compromised shoulder movements is questionable. In this study, only the muscle activity of the infraspinatus muscle was measured among the rotator cuff muscles; therefore, the results could not explain the changes in the muscle activity of the rotator cuff muscles. Follow-up studies should be conducted on study subjects with SIS and impaired movement of supraspinatus, teres minor, and subscapularis depending on the various positions and flexion angles of shoulder joints.

\section{CONCLUSION}

The muscle activity of the scapular upward rotators was significantly higher when the upper arm was rotated outward. The muscle activity of the infraspinatus and anterior deltoid muscles was also high. Therefore, in order to prevent SIS, it is recommended to perform in an external rotated position of the upper arm when exercising for strengthening the scapular upward rotators, rotator cuff muscles, and anterior deltoid muscles.

\section{Key Points}

Question What upper arm position and the shoulder flexion angle are effective for improvement on the scapular upward rotators, infraspinatus, and deltoid muscles?

Findings The muscle activities of the scapular upward rotators, infraspinatus and anterior deltoid muscles were significantly higher when the upper arm was rotated outward than the other humeral positions.

Meaning In order to prevent shoulder impingement syndrome, it is recommended to perform in an external rotated position of the upper arm when exercising for strengthening the scapular upward rotators, infraspinatus, and anterior deltoid muscles.

\section{Article information}

Conflict of Interest Disclosures: We wish to confirm that there are no known conflicts of interest associated with this publication.

Funding/Support: There has been no significant financial support for this work that could have influenced its outcome.

Acknowledgment: None.

Ethic Approval: All of the subjects provided informed consent. This study was approved by the Institutional Review Board of Joongbu University (JIRB-2017051901 -05-170612).

\section{REFERENCES}

1. Neer CS. Anterior acromioplasty for the chronic impingement syndrome in the shoulder: a preliminary report. JBJS. 1972;54(1):41-50.

2. Hallström E, Kärrholm J. Shoulder kinematics in 25 patients with impingement and 12 controls. Clin Orthop Relat R. 2006;448:22-27.

3. Ludewig PM, Cook TM. Translations of the humerus in persons with shoulder impingement symptoms. J Orthop Sports Phys Ther. 2002;32(6):248-259.

4. McClure PW, Michener LA, Karduna AR. Shoulder function and 3-dimensional scapular kinematics in people with and without shoulder impingement syndrome. Phys Ther. 2006;86(8):1075-1090.

5. Myers JB, Hwang JH, Pasquale MR, et al. Shoulder muscle coactivation alterations in patients with subacromial impingement. Med Sci in Sports Exerc. 2003;35: S346.

6. Warner JJ, Micheli LJ, Arslanian LE, et al. Patterns of flexibility, laxity, and strength in normal shoulders and shoulders with instability and impingement. Am J Sports Med. 1990;18(4):366-375.

7. Reinold MM, Macrina LC, Wilk KE, et al. Electromyographic analysis of the supraspinatus and deltoid muscles during 3 common rehabilitation exercises. J Athl Train. 2007;42(4):464.

8. Sahrmann S. Diagnosis and treatment of movement impairment syndromes. St Louis, MO: Mosby Inc; 2002.

9. Weon JH, Oh JS, Cynn HS, et al. Influence of forward head posture on scapular upward rotators during isometric shoulder flexion. J Bodyw Mov Ther. 2010;14(4):367374.

10. Ludewig PM, Cook TM. Alterations in shoulder kinematics and associated muscle activity in people with symptoms of shoulder impingement. Phys Ther. 2000; 80(3):276-291. 
11. Kendall FP, McCreary EK, Provance PG. Muscles: testing and function with posture and pain. 5th ed. Baltimore, MD: Lippincott Williams \& Wilkins; 2005.

12. Lin JJ, Lim HK, Yang JL. Effect of shoulder tightness on glenohumeral translation, scapular kinematics, and scapulohumeral rhythm in subjects with stiff shoulders. J Orthop Res. 2006;24(5):1044-1051.

13. Ludewig PM, Cook TM, Nawoczenski DA. Threedimensional scapular orientation and muscle activity at selected positions of humeral elevation. J Orthop Sports Phys Ther. 1996;24(2):57-65.

14. McClure PW, Bialker J, Neff N, et al. Shoulder function and 3-dimensional kinematics in people with shoulder impingement syndrome before and after a 6-week exercise program. Phys Ther. 2004;84(9):832-848.

15. Cools AM, Declercq GA, Cambier DC, et al. Trapezius activity and intramuscular balance during isokinetic exercise in overhead athletes with impingement symptoms. Scand J Med Sci Sports. 2007;17(1):25-33.

16. Cools AM, Witvrouw EE, Declercq GA, et al. Evaluation of isokinetic force production and associated muscle activity in the scapular rotators during a protraction-retraction movement in overhead athletes with impingement symptoms. Br J Sports Med. 2004; 38(1): 64-68.

17. Cools AM, Witvrouw EE, Declercq GA, et al. Scapular muscle recruitment patterns: trapezius muscle latency with and without impingement symptoms. Am J Sports Med. 2003;31(4):542-549.

18. Ludewig PM, Hoff MS, Osowski EE, et al. Relative balance of serratus anterior and upper trapezius muscle activity during push-up exercises. Am J Sports Med. 2004;32(2):484-493.

19. Arlotta M, LoVasco G, McLean L. Selective recruitment of the lower fibers of the trapezius muscle. J Electromyogr Kinesiol. 2011;21(3):403-410.

20. Decker MJ, Hintermeister RA, Faber KJ, et al. Serratus anterior muscle activity during selected rehabilitation exercises. Am J Sports Med. 1999;27(6):784-791.

21. Ekstrom RA, Donatelli RA, Soderberg GL. Surface electromyographic analysis of exercises for the trapezius and serratus anterior muscles. J Orthop Sports Phys Ther. 2003;33(5):247-258.

22. Bitter NL, Clisby EF, Jones MA, et al. Relative contributions of the infraspinatus and deltoid during external rotation in patients with symptomatic subacromial impingement. J Shoulder Elbow Surg. 2007;16(5): 563-568

23. Reinold MM, Wilk KE, Fleisig GS, et al. Electromyo- graphic analysis of the rotator cuff and deltoid musculature during common shoulder external rotation exercises. J Orthop Sports Phys Ther. 2004; 34(7):385-394.

24. Hutson M, Ward A. Oxford textbook of musculoskeletal medicine. Oxford University Press; 2015.

25. Weon JH, Kwon OY, Cynn HS, et al. Real-time visual feedback can be used to activate scapular upward rotators in people with scapular winging: an experimental study. J Physiother. 2011;57(2):101-107.

26. Antony NT, Keir PJ. Effects of posture, movement and hand load on shoulder muscle activity. $J$ Electromyogr Kinesiol. 2010;20(2):191-198.

27. MacDonell CW, Keir PJ. Interfering effects of the task demands of grip force and mental processing on isometric shoulder strength and muscle activity. Ergonomics. 2005;48(15):1749-1769.

28. Xavier RL, Paul A, Veronique G, et al. Shoulder coordination during full-can and empty-can rehabilitation exercises. J Athl Train. 2015;50(11):1117-1125.

29. Criswell E. Cram's introduction to surface electromyography. Jones and Bartlett Publishers; 2010.

30. Hermens HJ, Freriks B, Disselhorst-Klug C, et al. Development of recommendations for SEMG sensors and sensor placement procedures. J Electromyogr Kinesiol. 2000;10(5):361-374.

31. Moseley JB, Jobe FW, Pink M, et al. EMG analysis of the scapular muscles during a shoulder rehabilitation program. Am J Sports Med. 1992;20(2):128-134.

32. Rack PM, Westbury DR. The effects of length and stimulus rate on tension in the isometric cat soleus muscle. J Physiol. 1969;204(2):443-460.

33. Ebaugh DD, McClure PW, Karduna AR. Three-dimensional scapulothoracic motion during active and passive arm elevation. Clin Biomech. 2005;20(7):700-709.

34. Neumann DA. Kinesiology of the musculoskeletal system. St Louis, MO: Mosby; 2002.

35. Lehman GJ, MacMillan B, Maclntyre I, et al. Shoulder muscle EMG activity during push up variations on and off a Swiss ball. Dyn Med. 2006;5:7.

36. Lopes AD, Timmons MK, Grover M, et al. Visual scapular dyskinesis: kinematics and muscle activity alterations in patients with subacromial impingement syndrome. Arch Phys Med Rehabil. 2015;96(2):298-306.

37. Park KM, Cynn HS, Yi CH, et al. Effect of isometric horizontal abduction on pectoralis major and serratus anterior EMG activity during three exercises in subjects with scapular winging. J Electromyogr Kinesiol. 2013; 23(2):462-468.

38. Ha SM, Kwon OY, Cynn HS, et al. Comparison of 
electromyographic activity of the lower trapezius and serratus anterior muscle in different arm-lifting scapular posterior tilt exercises. Phys Ther Sport. 2012;13(4): 227-232. 